Metaphor Comprehension - A special mode of langliage processing? (Extended Abstract)

Jon M. Slack

Open University, U.K.

The paper addresses the question of whether a complete language understanding system requires special procedures in order to comprehend metaphorical language. To answer this question it is necessary to delineate the processes involved in metaphor comprehension and to determine the uniqueness of such processes in the context of existing language understanding systems.

\section{DEFINING THE PROBLEM}

For the gurposes of this paper a metaphor is defined as a linguistic input containing elements which result in a mismatch at the semantic level which the language understanding system attempts to interpret. For example, the sentence

Btllboards are warts on the landscape . 1

results in a semantic mismatch represented by the sentence

Billboards are not a member

of the category warts .... 2

which is encountered when the underlying conceptual-structure is built for the sentence. However, the mismatch need not be restricted to elements of the same sentence. Even though the elements of a particular sentence may not result in a semantic mismatch, the whole sentence itself can be metaphorical with respect to its linguistic context. In this case the seman tic mismatch is encountered when the interpreter has difficulty connecting the conceptual representation of the sentence to the existing structure representing the context.

Metaphor comprehension is defined as the process of mapping input metaphors onto connected conceptual representations.

A model of metaphor comprehension is defined as the set of processes required to interpret elements of linguistic input which semantically mismatch their linguistic context. The mismatching elements are referred to as the vehicle of the metaphor, while the linguistic context is known as the topic of the metaphor.

\section{BUILDING A MODEL OF METAPHOR COMPREHENSION}

For the purposes of describing the model only sentences which contain both the vehicle and topic elements of the metaphor are considered. However, the comprehension processes described should apply to all classes of metaphor, including copula form (e.g. The world is a chessboard) and verb-based metaphors (e.g. His words were dried by the sun). The model to be outlined is based on the analysis of a large sample of paraphrases produced by twenty subjects for a collection of over fifty metaphors.
It is necessary to distinguish between different types of comprehension task. Although the comprehension processes are generally applicable. whether the metaphorical input is in isolation or part of a larger linguistic input greatly influences the choice of comprehension strategy.

In line with most language comprehension systems, the goal of metaohor comprehension is to build an integrated conceptual structure but from mismatching components. The model is based on the notion that the existence of the semantic mismatch makes it necessary to build a conceptual structure which embodies all the salient knowledge structures associated with the vehicle element. This process is referred to as vehicle expansion.

Briefly, the comprehension process proceeds as follows :

The elements of the sentence are mapped onto their dictionary entries and in attempting to build a conceptual structure a semantic restriction violation (semantic mismatch) is encountered - the subject, or object, or both do not conform to the semantic restrictions associated with the verb. The decision of which element represents the topic and which the vehicle of the metaphor is usualiy determined by the extra-sentential context. However. for \pm solated metaphors the vehicle is usually the element which has the minimal match with the other elements. The knowledge structures which constitute the vehicle element concept are temporarily built into the conceptual structure representing the meaning of the sentence. This generated conceptual structure (CCS) is also connected to the topic knowledge structures (TKS). Comprehension is complete when the GCS and TKS are integrated into a single conceptual structure. Vehicle expansion generates a relatively large conceptual structure which is pruned by means of the processes which integrate the GCS and TKS. For example, those knowledge structures which gave rise to the semantic mismatch are deleted from the conceptual structure because they contradict what is known about the topic. Metaphor comprehension involves interpreting the GCS in terms of the TKS. This is achieved by means of a matching process and the comprehension strategies described below.

The matching process searches for matching know ledge structures in the GCS and TXS. Various forms of the process are considered - fuzzy matching procedures, a spreading activation process. The outcome of the process is typically, (a) an element of the GCS matches a lowsaliency TRS element, (b) an element of the cCS contradicts a TKS element, or (c) a GCS element matches nor contradicts any TKS element. Outcome (b) prunes the GCS; outcomes (a) and (c) connect it to the TKS. 
In addition, certain input metaphors require other comprehension strategies to be invoked, such as context construction or recursive metaphor interpretation.

Context construction - in some cases, although the matching procedure has constructed a number of important connections between the GCS and the TKS, it is necessary to search for additional knowledge structures which represent context information which was missing in the original input. The goal of the context construction procedure is to provide a context information in which the GCS-TKS connections are fully interpreted, that is, more fully integrated. The paper discusses the conditions necessary for the strategy to be employed.

Recursive metaphor interpretation - many of the elements of the GCS are themselves metaphors in that they contain implicit semantic mismatches. These metaphoric elements are interpreted as metaphor inputs thereby making the comprehension process recursive.

Due to the limited processing capacity and memory constraints of language understanding systems, the metaphor comprehension process requires a complex control structure. This control structure governs the use of the comprehension strategies and orders the vehicle expansion and matching processes.

\section{COMPARISON WITH OTHER THESRIES}

The paper compares the model of metaphor comprehension with established theorles of metaphor within linguisties and psychology.

\section{CONCLUSIONS}

The final part of the paper examines the relationship between metaphor comprehension and existing language comorehension systems. The processes which constitute the model of metaphor comprehension are common to many language understanding systems (Schank, Wilks, InR group, etc.). The comprehension of metaphor does not require a special set of processes to be developed, although the comprehension strategies may be specific to the comprehension task. Rather, a metaphor input forces the comprehension system to invoke a complex control structure to cope with the larger and richer knowledge domains which have to be handled. The main conclusion of the paper is that the notions of processing capacity. memory constraints and control structure are the most salient constructs in simulation metaphor comprehension. 